University of Nebraska - Lincoln

DigitalCommons@University of Nebraska - Lincoln

March 1986

\title{
Photoelectron angular distributions for atomic chlorine
}

\author{
Siamak Shahabi \\ University of Nebraska - Lincoln \\ Anthony F. Starace \\ University of Nebraska-Lincoln, astarace1@unl.edu
}

Follow this and additional works at: https://digitalcommons.unl.edu/physicsstarace

Part of the Physics Commons

Shahabi , Siamak and Starace, Anthony F., "Photoelectron angular distributions for atomic chlorine" (1986). Anthony F. Starace Publications. 29.

https://digitalcommons.unl.edu/physicsstarace/29

This Article is brought to you for free and open access by the Research Papers in Physics and Astronomy at DigitalCommons@University of Nebraska - Lincoln. It has been accepted for inclusion in Anthony F. Starace Publications by an authorized administrator of DigitalCommons@University of Nebraska - Lincoln. 


\title{
Photoelectron angular distributions for atomic chlorine
}

\author{
Siamak Shahabi* and Anthony F. Starace \\ Department of Physics and Astronomy, The University of Nebraska, Lincoln, Nebraska 68588-0111
}

(Received 3 September 1985)

\begin{abstract}
Photoelectron angular distribution asymmetry parameters for photoionization to each of the three term levels of the $\mathrm{Cl}^{+}$ion are calculated above the ${ }^{1} S$ ionic threshold with use of the open-shell transition-matrix method. Comparison is made with frozen-core Hartree-Fock asymmetry parameters and with the multiconfiguration Hartree-Fock results of Fielder and Armstrong. The calculated asymmetry parameters are used to estimate partial-cross-section branching ratios at $584 \AA$ from the relative experimental photoelectron intensity measurements of Kimura, Yamazaki, and Achiba.
\end{abstract}

\section{INTRODUCTION}

Atomic chlorine has served as the test for theoretical treatments of electron correlation effects on the photoionization cross section of open-shell atoms. ${ }^{1-7}$ One reason ${ }^{2}$ for the choice of chlorine is that it is adjacent to argon in the periodic table and thus could be compared with known results for argon, which has served as the test for theories of closed-shell atom photoionization processes. ${ }^{8}$ However, the same theoretical methods which produce results in good agreement for photoionization of the $3 p$ subshell of argon ${ }^{8}$ produce disparate results for the photoionization of the $3 p$ subshell of atomic chlorine. ${ }^{1-7}$

The only experimental cross-section measurements above the ${ }^{1} S$ ionization threshold, the region on which the theoretical calculations have focused, are the relative total photoionization cross-section data of Samson, Shafer, and Angel. ${ }^{9}$ These are in good qualitative agreement with four $^{3,4,6,7}$ of the theoretical predictions below $500 \AA$ (i.e., $\hbar \omega \geqq 0.91$ a.u.). More recently these data have been put on an absolute basis with error bars of $\pm 10 \% .{ }^{10}$ They lie between 0 and $12 \%$ below these four theoretical results in the region below $500 \AA$. Between the ${ }^{1} S$ threshold and about $530 \AA$ the experimental data lie significantly above all the most recent theoretical predictions. ${ }^{3-7}$ The rise in the total cross section near the ${ }^{1} S$ threshold predicted by two of the calculations ${ }^{6,7}$ is not found experimentally. ${ }^{10}$

While the excellent quantitative agreement of the absolute experimental total cross section ${ }^{10}$ with four of the recent theoretical calculations below $500 \AA$ is gratifying, the sizable quantitative discrepancies between the absolute experimental data ${ }^{10}$ and these calculations above the ${ }^{1} S$ threshold are difficult to interpret at this time. Clearly further comparisons among the alternative theoretical predictions as well as with additional experimental data are necessary to understand those electron correlations which affect the gross oscillator strength distribution in openshell atoms such as atomic chlorine.

This Brief Report extends the computational evidence on the photoionization of the $3 p$ subshell of atomic chlorine above the ${ }^{1} S$ threshold. We present calculations of the photoelectron angular distributions for each of the three term levels (i.e., ${ }^{3} P,{ }^{1} D$, and ${ }^{1} S$ ) of $\mathrm{Cl}^{+}$using our recently developed open-shell transition matrix (OSTM) method. ${ }^{11}$ This report is an addendum to our recent OSTM calculations of the total and partial cross sections for the $3 p$ subshell of atomic chlorine. ${ }^{7}$ Independent particle model [i.e., Hartree-Fock (HF)] calculations of the angular distributions for atomic chlorine have been published, employing both unrelaxed ${ }^{2}$ and relaxed ${ }^{12}$ ionic core wave functions. Of the calculations which include the important final-state interchannel interactions, ${ }^{1,3,4,5,6}$ however, only those of Fielder and Armstrong ${ }^{6}$ predict photoelectron angular distributions. While our OSTM angular distribution results agree qualitatively with theirs, the quantitative differences are substantially greater than is the case for the total cross section.

\section{THEORY}

We describe the photoelectron angular distribution in terms of the well-known asymmetry parameter, $\beta,{ }^{13}$ using the angular momentum transfer formulation of Dill and Fano. ${ }^{14}$ The form of the angular momentum transfer formulation appropriate in $L S$ coupling has been reviewed recently by Manson and Starace ${ }^{15}$ and the form of the dipole amplitudes in the OSTM method has been presented elsewhere. ${ }^{7,11}$ While a self-contained description of the theory is not possible in this Brief Report, we do endeavor here to provide detailed references to these earlier works so that the interested reader may reconstruct the formulas employed in our calculations.

The asymmetry parameters for each of the three term levels of $\mathrm{Cl}^{+}$may be expressed in terms of scattering amplitudes, $S_{l}\left(j_{t}\right)$, where $l$ is the photoelectron orbital angular momentum and $j_{t}$ is the angular momentum transfer, as follows: ${ }^{16}$

$$
\begin{aligned}
& \beta\left({ }^{1} S\right)=N / D, \\
& \beta\left({ }^{1} D\right)=\frac{N-5\left|S_{2}(2)\right|^{2}+2\left|S_{2}(3)\right|^{2}}{D+5\left|S_{2}(2)\right|^{2}+7\left|S_{2}(3)\right|^{2}}, \\
& \beta\left({ }^{3} P\right)=\left[N-5\left|S_{2}(2)\right|^{2}\right] /\left[D+5\left|S_{2}(2)\right|^{2}\right],
\end{aligned}
$$

where 


$$
\begin{aligned}
& N \equiv 3\left|S_{2}(1)\right|^{2}-3 \times 2^{1 / 2}\left[S_{2}(1) S_{0}^{\dagger}(1)+S_{2}(1)^{\dagger} S_{0}(1)\right] \\
& D \equiv 3\left|S_{0}(1)\right|^{2}+3\left|S_{2}(1)\right|^{2}
\end{aligned}
$$

In these equations, the scattering amplitudes $S_{l}\left(j_{t}\right)$, and hence the expressions for $N$ and $D$, are implicitly dependent on the ionic term level. Their precise expression in $L S$ coupling in terms of incoming-wave-normalized dipole amplitudes is given by Eq. (3.10) of Ref. 15.

The equations above, together with Eq. (3.10) of Ref. 15 , describe the geometrical constraints on the asymmetry parameter assuming only that $L S$ coupling is valid; the dynamical information is contained in the incomingwave-normalized dipole amplitudes. The OSTM expression for the incoming-wave-normalized dipole amplitudes is given by Eq. (14) of Ref. 7(a) in terms of the standingwave-normalized dipole amplitudes defined by Eqs. (12)-(13) therein. The angular factors multiplying the reduced dipole matrix elements in Eq. (12) of Ref. 7(a) are defined by Eq. (18) of Ref. 11. ${ }^{17}$

\section{RESULTS AND DISCUSSION}

As was the case for the total and partial cross sections, final-state interchannel interactions dominate the photoelectron angular distribution in atomic chlorine. The frozen-core HF $\beta$ parameters of Starace and Armstrong ${ }^{2}$ differ from the relaxed core HF $\beta$ parameters of Manson, Msezane, Starace, and Shahabi ${ }^{12}$ primarily by an energy shift. The effect of virtual pair excitations out of the $3 p$ subshell on the asymmetry parameters in the absence of final-state interchannel coupling has been shown to be small. $^{2}$ Because these effects are small in comparison with those of final-state interchannel coupling, we compare the present OSTM $\beta$ parameters only with our frozen core HF results and with the multiconfiguration HartreeFock (MCHF) results of Fielder and Armstrong. ${ }^{6}$

Figures 1-3 show the photoelectron asymmetry parameters $\beta$ for each of the three final ionic term levels, ${ }^{3} P,{ }^{1} D$, and ${ }^{1} S$, of the chlorine ion. For the ${ }^{3} P$ term level the HF results are dome-shaped, the OSTM results are flat-topped and of lower magnitude, and the MCHF results are increasing and of still lower magnitude until an energy of about 0.9 a.u. Above 0.9 a.u. the HF $\beta$ decreases fastest and the OSTM and MCHF results appear to be converging toward one another as they decrease.

For the ${ }^{1} D$ term level, the shapes appear similar to those for the ${ }^{3} P$ case: the HF curves are dome-shaped, the OSTM curves are much flatter, and the MCHF curves are slightly increasing. Above $0.8 \mathrm{eV}$ the $\mathrm{HF} \beta$ curve decreases fastest and the OSTM and MCHF velocity curve tend to converge as they decrease. In contrast to the ${ }^{3} P$ case, however, the OSTM $\beta$ values are substantially lower than the HF $\beta$ values over the region from threshold to 0.7 a.u. above. The MCHF $\beta$ values are lower still.

For the ${ }^{1} S$ term level, the shapes of the HF, OSTM, and MCHF are similar to those for the ${ }^{3} P$ and ${ }^{1} D$ cases up to about 0.6 a.u. with the exception that the OSTM $\beta$ has an

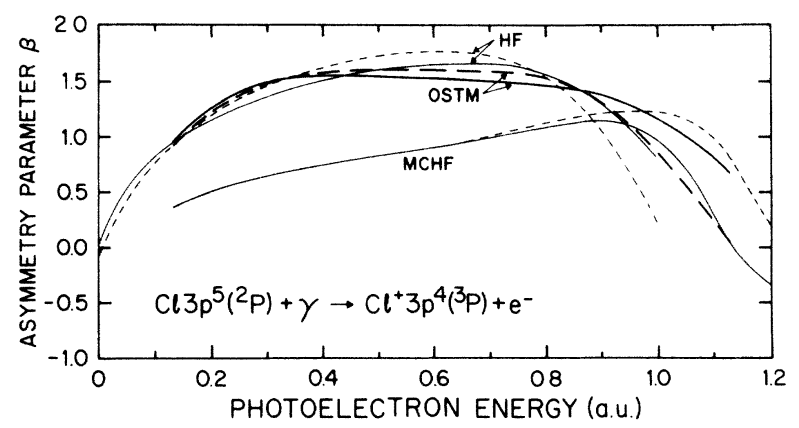

FIG. 1. Photoelectron asymmetry parameter $\beta$ for photoionization of the ground state of atomic chlorine leading to the ${ }^{3} P$ state of the chlorine ion plotted vs photoelectron energy in a.u. The solid and dashed curves, respectively, represent results using the length and velocity electric dipole formulas. HF; present frozen-core Hartree-Fock results. OSTM; present open-shell transition matrix results. MCHF; multiconfiguration HartreeFock results of Fielder and Armstrong (Ref. 6).

even sharper increase above threshold than does the HF $\beta$. Above 0.6 a.u., however, the OSTM curve exhibits a peak that is only hinted at in the MCHF $\beta$ curves [but which is consistent with other, less detailed MCHF predictions for $\beta$ (Ref. 18)].

Our conclusions on the effect of final-state interchannel interactions on the $\beta$ parameters are as follows. Both the OSTM and the MCHF calculations predict that the $\beta$ parameter is flatter as a function of energy immediately above threshold, not increasing to as high values as found in HF calculations. Furthermore, both the OSTM and MCHF calculations predict that the $\beta$ parameter decrease due to the Cooper minimum in the $3 p \rightarrow d$ dipole amplitude, discussed elsewhere, ${ }^{12}$ occurs at higher photoelectron energies. The MCHF predictions differ from the OSTM ones quantitatively, however: The MCHF $\beta$ values are lower than those predicted by the OSTM calculations presented here, i.e., the MCHF calculations predict more isotropic angular distributions than are predicted by the OSTM calculations.

There are two significant differences between the OSTM and MCHF calculations which may explain the

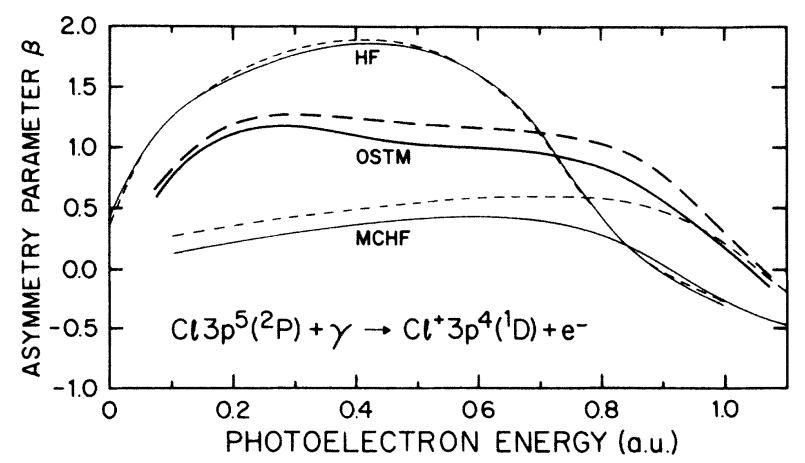

FIG. 2. Photoelectron asymmetry parameter $\beta$ for photoionization of $\mathrm{Cl}$ leading to the ${ }^{1} \mathrm{D}$ state of $\mathrm{Cl}^{+}$. Same notation as for Fig. 1. 


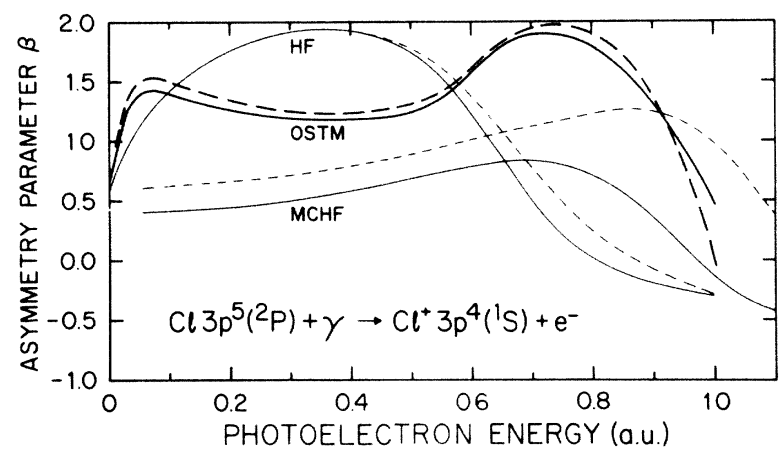

FIG. 3. Photoelectron asymmetry parameter $\beta$ for photoionization of $\mathrm{Cl}$ leading to the ${ }^{1} S$ state of $\mathrm{Cl}^{+}$. Same notation as for Fig. 1.

quantitative differences between their predicted $\beta$ parameters. Firstly, the MCHF calculations do not include interchannel interactions between the $3 p \rightarrow \epsilon s$ channels and the $3 p \rightarrow \epsilon d$ channels except in the case of ${ }^{2} S$ final-state term levels. The OSTM calculations include all final-state interchannel interactions. Secondly, the MCHF calculations include effects of virtual pair excitations out of both the $3 s$ and $3 p$ subshells while the OSTM calculations in- clude virtual pair excitations out of the $3 p$ subshell only. Given that the effects of virtual pair excitations out of the $3 p$ subshell on the cross sections and $\beta$ parameters have been shown to be small, ${ }^{2,7}$ it is probable that the neglect of interchannel coupling of the final-state $d$ and $s$ channels in the MCHF calculations is responsible for a large part of the quantitative difference between the MCHF and the present OSTM $\beta$ parameter predictions.

Finally, it is of interest to reconsider the correction of the relative experimental photoelectron intensity measurements of Kimura, Yamazaki, and Achiba ${ }^{19}$ at $584 \AA$ due to photoelectron angular distribution effects. In our previous work ${ }^{7}$ this was done using the $\beta$ parameters predicted by Fielder and Armstrong. ${ }^{\circ}$ Using the average of our present OSTM length and velocity results we find that at $584 \AA, \quad \beta\left({ }^{3} P\right)=1.50, \quad \beta\left({ }^{1} D\right)=1.23$, and $\beta\left({ }^{1} S\right)=1.32$. Those $\beta$ values imply ${ }^{7}$ that the uncorrected ${ }^{1} D:{ }^{3} P$ branching ratio ${ }^{19}$ should be increased by $5.1 \%$ to 0.85 and the uncorrected ${ }^{1} S:{ }^{3} P$ branching ratio should be increased by $3.4 \%$ to 0.165 . These compare with the $8 \%$ and $4 \%$ corrections found ${ }^{7}$ using the $\beta$ parameters of Ref. 6 .

\section{ACKNOWLEDGMENT}

This work was supported in part by the National Science Foundation under Grant No. PHY-83-05739.
*Present address: Division of Radiation Oncology, Wisconsin Clinical Cancer Center, University of Wisconsin Hospitals, 600 Highland Avenue, Madison, WI 53792.

${ }^{1}$ (a) M. J. Conneely, Ph.D. thesis, London University, 1969 (unpublished); (b) M. J. Conneely, K. Smith, and L. Lipsky, J. Phys. B 3, 493 (1970).

${ }^{2}$ A. F. Starace and L. Armstrong, Jr., Phys. Rev. A 13, 1850 (1976).

3(a) N. A. Cherepkov and L. V. Chernysheva, Phys. Lett. 60A, 103 (1977); (b) Izv. Akad. Nauk SSSR, Ser. Fiz. 41, 2518 (1977) [Bull. Acad. Sci. USSR, Phys. Ser. 41, 47 (1977)].

${ }^{4}$ E. R. Brown, S. L. Carter, and H. P. Kelly, Phys. Lett. 66A, 290 (1978); Phys. Rev. A 21, 1237 (1980).

${ }^{5} \mathrm{M}$. Lamoureux and F. Combet Farnoux, J. Phys. (Paris) 40, 545 (1979).

6(a) W. R. Fielder and L. Armstrong, Jr., Phys. Rev. A 28, 218 (1983); (b) William Reynolds Fielder, Ph.D. thesis, Johns Hopkins University, 1981 (unpublished) (available through University Microfilms International, 300 N. Zeeb Road, Ann Arbor, Michigan 48106, Order No. 8106608).

${ }^{7}$ (a) S. Shahabi, A. F. Starace, and T. N. Chang, Phys. Rev. A 30, 1819 (1984). (b) Siamak Shahabi, Ph.D. thesis, The University of Nebraska, 1983 (unpublished) (available through University Microfilms International, 300 N. Zeeb Road, Ann Arbor, Michigan 48106, Order No. DA8328194).

${ }^{8}$ A. F. Starace, in Handbuch der Physik, edited by W. Mehlhorn (Springer, Berlin, 1982), Vol. 31, pp. 1-121. See especially Chap. III and references therein.

9 J. A. R. Samson, Y. Shafer, and G. C. Angel, in Electronic and
Atomic Collisons: Abstracts of Contributed Papers, edited by M. J. Coggiola, D. L. Huestis, and R. P. Saxon (XIV ICPEAC, Palo Alto, CA, 1985), Abstract H6, p. 6.

10J. A. R. Samson and G. C. Angel (private communication).

${ }^{11}$ A. F. Starace and S. Shahabi, Phys. Rev. A 25, 2135 (1982).

${ }^{12}$ S. T. Manson, A. Msezane, A. F. Starace, and S. Shahabi, Phys. Rev. A 20, 1005 (1979).

${ }^{13}$ J. Cooper and R. N. Zare, in Lectures in Theoretical Physics, edited by S. Geltman, K. T. Mahanthappa, and W. E. Britten (Gordon and Breach, New York, 1969), Vol. XI-C, pp. $317-337$.

${ }^{14}$ D. Dill and U. Fano, Phys. Rev. Lett. 29, 1203 (1972); U. Fano and D. Dill, Phys. Rev. A 6, 185 (1972); D. Dill ibid. 7, 1976 (1973).

${ }^{15}$ S. T. Manson, and A. F. Starace, Rev. Mod. Phys. 54, 389 (1982).

${ }^{16}$ Equations (1a)-(1e) follow from Eqs. (3.8)-(3.9) of Ref. 15 after a trivial calculation of the allowed values of $j_{t}$ from Eq. (3.5).

${ }^{17}$ Note that both Eqs. (18b) and (18c) of Ref. 11 are missing a phase factor $(-1)^{L_{i}+L_{f}}$. This factor is unnecessary for calculating the total and partial cross sections presented in Ref. 7. It is essential in calculating the photoelectron asymmetry parameters presented here due to the interference of dipole amplitudes with alternative final total angular momenta, $L_{f}$.

${ }^{18}$ See Fig. 35 on pp. $375-376$ of Ref. 6(b).

${ }^{19}$ K. Kimura, T. Yamazaki, and Y. Achiba, Chem. Phys. Lett. 58, 104 (1978). 